\title{
High mini nutritional assessment (MNA) correlates with bone ultrasound measurements in Swiss women $>75$ years of age who have a lifetime fracture history
}

\author{
Emma Wynn ${ }^{1}$, Marc-Antoine Krieg ${ }^{2}$, Peter Burckhardt ${ }^{1}$ and Susan Lanham-New ${ }^{3}$ \\ ${ }^{1}$ University Hospital, Lausanne, Switzerland, ${ }^{2}$ Bois-Cerf Clinic, Lausanne, Switzerland and ${ }^{3}$ University of Surrey, \\ Guildford, UK
}

To achieve optimum bone health, numerous factors are required such as optimal genetics, good health, weight-bearing physical activity and adequate nutrition ${ }^{(1)}$. Malnutrition is a risk factor for osteoporotic fractures and is a very common problem in the elderly population that is often under-diagnosed ${ }^{(2)}$. The $\mathrm{MNA}^{(3)}$ is an effective easily-administered tool designed to identify older adults who have or are at risk for developing malnutrition. It consists of eighteen questions and can be completed in about $15 \mathrm{~min}$.

The aim of the study was to assess the possible relationship between MNA and bone ultrasound (QUS) measurements at the heel (broadband ultrasound attenuation; BUA). A trend for a correlation between the MNA and BUA has already been reported in a previous study ${ }^{(4)}$ but the intention was to explore this association further in a very-elderly group of the Swiss population who had reported a previous fracture. Furthermore, a more recent study has shown that elderly free-living women who have an MNA score $<27$ have a twofold increased risk of having osteoporosis ${ }^{(5)}$.

As part of an ongoing study (EVANIBUS) on the evaluation of nutritional intake and bone ultrasound ${ }^{(6)}$ MNA and QUS were assessed in 256 elderly Swiss ambulatory women who had reported a fracture in their lifetime (age 80.6 (SD 3.0) years, BMI 24.6 (SD 4.4) kg/m², BUA 96.8 (SD 9.0) dB/mHz). QUS, carried out using an Achilles ultrasound densitometer, (Lunar Corporation, Madison, WI, USA), is predictive of fracture risk in elderly women. The MNA, a non-invasive and validated questionnaire to evaluate nutritional status in the elderly, was completed by each subject. The MNA, which comprises eighteen questions, evaluates the nutritional status of the subject. The score range is $0-30(<17$, malnutrition; 17.5-23.5, risk of malnutrition; $\geq 24$, well-nourished).

The mean MNA score was 26.5 (SD 2.5). A higher MNA score was significantly associated with higher BUA $(r 0.149 ; P<0.05)$. BUA increased significantly between MNA quartile (Q) 1 and MNA Q4 (Q1, 23.5 (SD 2.1); Q2 26.5 (SD 2.4); Q3, 28 (SD 2.1); Q4, 30 (SD 2.2)). The difference among the mean scores for BUA for the four groups was assessed using a one-way ANOVA post-hoc test $(P=0.004)$ as well as $\mathrm{F}$ test linearity $(P=0.015)$. BUA values were significantly lower when the MNA score was $<24$ (risk of malnutrition). A high MNA score was correlated with higher BUA values in women who had suffered fractures, but the effect of the association was relatively low compared with that of age and BMI.

In hospitals a low MNA score is associated with an increase in mortality, longer length of hospitalisation and higher risk of going into a nursing home at discharge. The MNA has been shown to detect risk of malnutrition before changes are apparent in weight or serum proteins. The MNA is also a follow-up assessment tool. It should be included in geriatric assessment and has the advantage of being a cheap and easily-used tool. In addition, the MNA appears to be a useful tool for the evaluation of patients with osteoporosis, and further research is required to assess the role of the MNA in these patients.

1. Department of Health (1991) Dietary Reference Values for Food, Energy and Nutrients for the United Kingdom. Report on Health and Social Subjects no. 41. London. H. M. Stationery Office.

2. Food and Nutrition Board, Institute of Medicine (1997) Dietary Reference Intakes: For Calcium, Phosphorus, Magnesium, Vitamin D, and Fluoride. Washington, DC: National Academy Press.

3. Guigoz Y \& Vellas B (1999) Nestle Nutr Workshop Ser Clin Perform Programme 1, 3-11; discussion 11-2.

4. Gerber V, Krieg MA, Cornuz J, Guigoz Y \& Burckhardt P (2003) J Nutr Health Aging 7, 140-145.

5. Salminen H, Saaf M, Johansson SE, Ringertz H \& Strender LE (2006) Eur J Clin Nutr 60, 486-493

6. Dumartheray EW, Krieg MA, Cornuz J, Whittamore DR, Lanham-New SA \& Burckhardt P (2006) J Hum Nutr Diet 19, 431-435. 